have influenced the result. Recently, a new snare for the respiratory tract has become available, and a similar operation was successfully done more easily in similar a case (patient 1).

Although electrosurgery with a fiberoptic bronchoscope and a snare has some limitations, it is very useful and may be the first choice for the resection of polypoid tumors in the bronchial tree.

We are grateful to Ms. Chieko Yoshida, Mr. Toshihiko Kanno, Ms. Mieko Kosuga, and Ms. Mutsuko Izumi for their technical assistance.

\section{REFERENCES}

1. Jacobson MJ, LoCicero J. Endobronchial treatment of lung carcinoma. Chest 1991;100:837-41.

2. Marasso A, Gallo E, Massaglia GM, Onoscuri M, Bernardi V. Cryosurgery in bronchoscopic treatment of tracheobronchial stenosis. Chest 1993;103:472-4.

3. Petrou M, Goldstraw P. The management of tracheobronchial obstruction: a review of endoscopic technique. Eur J Cardiothorac Surg 1994;8:436-41.

4. Themelin D, Duchatelet P, Boudaka W, Lamy V. Endoscopic resection of an endobronchial hypernephroma metastasis using polypectomy snare. Eur Respir J 1990;3:732-3.

5. Gerasin VA, Shafirovsky BB. Endoscopic electrosurgery. Chest 1988;93:270-4.

\title{
CATAMENIAL PNEUMOTHORAX CAUSED BY DIAPHRAGMATIC ENDOMETRIOSIS
}

S. Blanco, PhD, F. Hernando, PhD, A. Gómez, PhD, M. J. González, MD, A. J. Torres, PhD, and J. L. Balibrea, PhD, Madrid, Spain

Catamenial pneumothorax is a recurrent syndrome of pneumothorax that occurs between 48 and 72 hours after menstruation. This syndrome was described for the first time in $1958^{1}$ and was named "catamenial pneumothorax" by Lillington in 1972. Since then more than 100 cases have been described in the literature. ${ }^{2}$ Considered a rare complication of systemic endometriosis, catamenial pneumothorax has been managed with a wide range of treatments ranging from thoracotomy with abrasive or chemical pleurodesis ${ }^{2}$ to the present-day treatment with oral contraceptives, progesterone, or gonadotropin-releasing hormone analogs. ${ }^{3}$

The causes of catamenial pneumothorax are diverse. Although most episodes are associated with diaphragmatic endometriosis, few have been confirmed in anatomopathologic studies. ${ }^{2,4,5}$ This report presents a case of catamenial pneumothorax in which systemic endometriosis was confirmed histologically.

Clinical summary. A 33-year-old woman had a history of chest pain associated with menstruation. In November 1992 she had an episode of right-sided pneumothorax that resolved with rest. In September 1995, she had chest pain on the right side with sudden dyspnea. Chest radiography revealed hydropneumothorax with complete collapse of the

From the Department of Surgery, Thoracic Surgery, Hospital Clinico "San Carlos," Madrid, Spain.

Received for publication Dec. 9, 1997; accepted for publication Jan. 9, 1998.

Address for reprints: S. Blanco, MD, Cirugia II y Cirugia Toracica, Hospital Clinico "San Carlos," C/Martin Lagos S/N, 28040, Madrid, Spain.

J Thorac Cardiovasc Surg 1998;116:179-80

Copyright (c) 1998 by Mosby, Inc.

0022-5223/98 $\$ 5.00+0 \quad \mathbf{1 2 / 5 4 / 8 8 8 1 9}$ lung. Endothoracic drainage resulted in complete reexpansion of the lung. On thoracic computed tomography no bullae were observed with pleural nodular swelling. Nuclear magnetic resonance imaging revealed an image in the pleura in the posterior arches of the fifth and sixth ribs of the right hemithorax, which could correspond to a blood focus or endometriosis. Abdominal ultrasonography did not reveal any pathologic findings. Intervention with videothoracoscopy was elected and bullae were detected in the apical segment of the upper lobe and in the posterior segment of the right lower lobe, which were resected. Mechanical pleural abrasion was performed. Exploration of the rest of the thoracic cavity revealed no associated lesions. Anatomopathologic study gave a diagnosis of bullous emphysema. In conjunction with the next menstrual cycle the patient again had severe chest pain on the right side and pleuritic signs. Chest radiography revealed apical and basal pneumothorax. On suspicion of catamenial pneumothorax a right axillary thoracotomy was performed in November 1995 with resection of several suggestive lesions from all the lobes and of a purple lesion $0.5 \mathrm{~cm}$ diameter from the right diaphragmatic cupula. Partial pleurectomy and pleural abrasion were again performed. Definitive anatomopathologic study revealed diaphragmatic endometriosis (Fig. 1). The patient was treated with gonadotropin-releasing hormone analogs because she wants to have children in the future. She is currently free of symptoms after 12 months of follow-up.

Discussion. Catamenial pneumothorax occurring in the few hours after menstruation accounts for $2.8 \%$ to $5.6 \%$ of all episodes of spontaneous pneumothorax in women. Usually numerous episodes of pneumothorax are necessary before the condition can be diagnosed. ${ }^{2}$ Most women affected with catamenial pneumothorax are in the third or fourth decade of their lives ${ }^{2,4,5}$ and the right hemithorax is affected in $90 \%$ to $95 \%$ of cases. ${ }^{2,4,5}$ The most frequent 


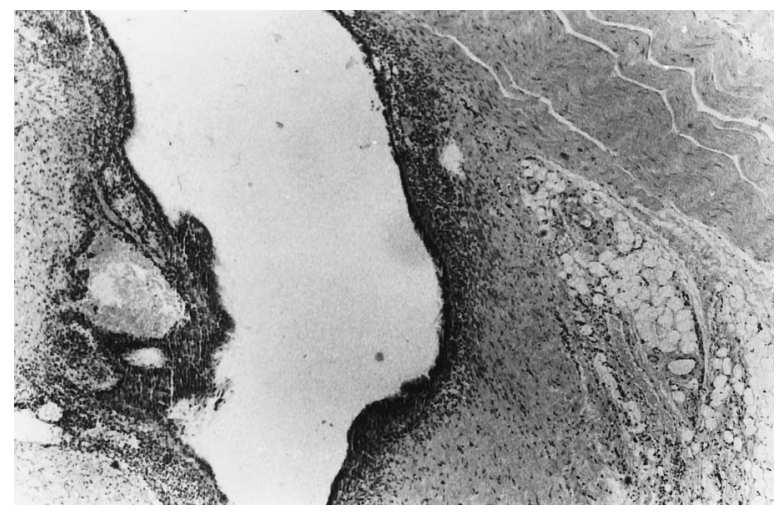

Fig. 1. Anatomopathologic study: Columnar epithelium resembling endometrial glands. Perforations in the columnar epithelium of cuboid cells similar to mesothelial cells are observed. The perforations are surrounded by fibrous tissue (hematoxylin and eosin; original magnification $\times 5$ ).

symptoms are chest pain or dyspnea, or both, on moderate exertion, although some patients are free of symptoms. ${ }^{2}$ Several theories on the pathogenesis of this entity have been suggested: (1) loss of cervical mucus during the menstrual phase assisting movement of air into the peritoneum, through the fenestra, mainly on the right side; (2) elevated platelet factor $2 \alpha$ in the serum during menstruation, which could cause severe vascular and bronchoalveolar constriction producing alveolar damage followed by an outlet of air to the pleural space; and (3) the theory that best fits the pathologic evidence, the existence of endometrial ectopic tissue in the visceral pleura and its desquamation during menstruation. ${ }^{2,4,5}$

Clinical or pathologic evidence of pelvic endometriosis is demonstrated in $30 \%$ to $60 \%$ of cases. In thoracotomies, pleural and/or diaphragmatic endometrial implants are observed in $30 \%$ to $50 \%$ of cases. ${ }^{2,4,5}$
An episode of catamenial pneumothorax is managed in exactly the same manner as other types of pneumothorax. When the patient is free of symptoms, she is treated conservatively. If the pneumothorax is greater than $20 \%$ with important symptoms, endothoracic drainage is carried out and analgesics are prescribed. A variety of options for long-term treatment exist, such as oral contraceptives to suspend ovulation, although relapses sometimes occur after this treatment, bilateral oophorectomy, danazol, and in recent years gonadotropin-releasing hormone analogs, which have been shown to give the best long-term results. ${ }^{3}$

On the other hand, from the first cases described, surgical treatment involving the creation of a strong pleural symphysis that impedes the recurrence of pneumothorax via a thoracotomy and mechanical or chemical pleural abrasion or pleurectomy have been carried out. $^{2,4,5}$ We therefore recommend that after pleural symphysis a therapeutic regimen of gonadotropin-releasing hormone analogs should be implemented ${ }^{3}$ to prevent new episodes of pneumothorax, especially in patients who wish to preserve their reproductive potential.

\section{REFERENCES}

1. Mauer ER, Schall JA, Mendez FL. Chronic recurring spontaneous pneumothorax due to endometriosis of the diaphragm. JAMA 1958;168:2013-4.

2. Shiraishi T. Catamenial pneumothorax: report of a case and review of the Japanese and non-Japanese literature. Thorac Cardiovasc Surg 1991;39:304-7.

3. Garris PD, Sokol MS, Kelly K, Whitman GF, Plouffre L. Leuprolide acetate treatment of catamenial pneumothorax: fertility and sterility. 1994;61:173-4.

4. Van-Schil PE, Vercauteren SR, Vermeire PA, Nackaerts YH, Van-Marck EA. Catamenial pneumothorax caused by thoracic endometriosis. Ann Thorac Surg 1996;62:585-6.

5. Yamashita J, Iwasaki A, Kawahara K, Shirakusa T. Thoracoscopic approach to the diagnosis and treatment of diaphragmatic disorder. Surg Laparosc Endosc 1996;6:485-8. 